\title{
On the Use of Fourier Methods in URC Fuzzy System Design
}

\author{
Jeffrey J. Weinschenk \\ University of Washington \\ Dept. of Electrical Engineering \\ Campus Box 352500 \\ Seattle, WA 98195-2500 \\ jjw77@ee.washington.edu
}

\author{
Robert J. Marks \\ Baylor University \\ Dept. of Engineering \\ Waco, Texas 76798 \\ r.marks@ieee.org
}

\author{
William E. Combs \\ The Boeing Company \\ P.O. Box 3707 \\ MS: $6 \mathrm{~F}-13$ \\ Seattle, WA 98124 \\ william.e.combs@boeing.com
}

\begin{abstract}
Previously, we demonstrated that rule explosion is avoidable in fuzzy inference engines by mapping an intersection rule configuration (IRC) to a union rule configuration (URC). Although conversion between IRC and URC systems is simple for a special, additively separable class of IRC rule tables, the conversion process is more difficult when the IRC rule table is additively inseparable. In a previous paper, we demonstrate a layered URC architecture can implement these types of systems, but did not provide a method for computing the weights. In this follow-on paper, we provide a Fourier-based algorithm for computation of the weights. We also prove that this technique is optimal in the sense that the mean-square approximation error is minimized for a fixed amount of resources. Finally, through the course of an example, we explore implementation issues and evaluate the overall effectiveness of the technique.
\end{abstract}

\section{INTRODUCTION}

$\mathrm{P}$ revious work has demonstrated that rule explosion is avoidable in fuzzy inference engines by converting an intersection rule configuration (IRC) to a union rule configuration (URC). Specifically, in [1-3], a mapping between the IRC and the URC is provided for a special class of IRC rule tables. In [2], a layered URC architecture is introduced that provides for universal approximation, and hence implementation of all other IRC rule tables. Currently, however, design of a layered URC system requires the use of costly (and potentially exhaustive) search techniques to find those projections that best represent a surface interpolated from the desired (IRC) rule table.

Here we provide a novel Fourier-based technique for designing layered URC fuzzy systems that eliminates the need for costly search techniques. Further, we prove that this technique is optimal in that the mean-square approximation error is minimized for a fixed amount of resources. Indeed, the mean-square approximation error can be reduced to an arbitrary degree such that relatively simple additively inseparable surfaces are reconstructed to a high degree of accuracy with a minimal cost in resources.

This paper is structured as follows: a brief review of the IRC, URC, and pertinent previous results are discussed in Section II. Section III follows with the development of the novel technique for URC fuzzy system design. A short example in Section IV illustrates the effectiveness of this technique and addresses various implementation issues. Section V closes with a few concluding remarks.

\section{A REVIEW OF THE IRC AND URC}

In [4], Combs differentiates between two types of fuzzy systems. In the first type, multi-antecedent rules map antecedent subsets connected with the intersection operator to a consequent subset. He refers to this structure as an intersection rule configuration (IRC). He refers to a second type of fuzzy system structure, which is composed strictly of single antecedent rules connected by the union operator, as a union rule configuration (URC) ${ }^{1}$.

The connection between these rule structures is conceptually well-represented in the propositional logic equivalence

$$
[(p \cap q) \Rightarrow r] \Leftrightarrow[(p \Rightarrow r) \cup(q \Rightarrow r)],
$$

where $p$ and $q$ are antecedents, $r$ is a consequent, $\cap$ represents intersection, $\cup$ represents union, and $\Rightarrow$ represents the implication operator. The expression on the left is indicative of an IRC, whereas the expression on the right is indicative of a URC. Importantly, the URC requires exponentially fewer rules than the IRC; hence, rule explosion is avoidable through use of a URC. However, in fuzzy logic, the IRC and the (single layer) URC rule structures are only equivalent under certain constraints [1-3,6-13]

Consider a multiple input, single output IRC fuzzy system in which the T-norm is implemented as a bounded sum, the T-conorm is implemented as a scalar product, and the implication operator is implemented as a scalar product (Mamdani implication). For $P$ antecedents and centroid defuzzification, the MISO IRC output formula is expressed in tensor ${ }^{2}$ form as,

\footnotetext{
${ }^{1} \mathrm{Yi}$ et al. propose a similar rule structure, which is composed of single input rule modules (SIRMs) [5]. However, under certain constraints, this structure is identical to the URC.

${ }^{2}$ In this paper, tensor notation adopted as it greatly simplifies the formulae that result.
} 


$$
y_{I R C}(\mathbf{x})=f_{a_{1}, a_{2}, \cdots, a_{P}} \prod_{i=1}^{P} \hat{\mu}_{i, a_{i}}\left(x_{i}\right),
$$

where $\hat{\mu}_{i, a_{i}}\left(x_{i}\right)$ is an element of a vector of sum-normalized membership values (one value per antecedent subset) for the $i^{\text {th }}$ antecedent. Each antecedent contains $N_{i}$ subsets, each of which are indexed by $a_{i}$. F denotes a $P^{\text {th }}$ order rule table tensor, an element of which is denoted $f_{a_{1}, a_{2}, \cdots, a_{P}}$. Since each consequent subset is completely characterized by its center of mass, each $f_{a_{1}, a_{2}}, \cdots, a_{P}$ is a scalar-valued center of mass.

In contrast, the output formula for a MISO URC fuzzy system with centroid defuzzification, using the same T-norm, T-conorm, and implication operators as defined above, is given by

$$
y_{U R C}(\mathbf{x})=\frac{1}{P} \sum_{i=1}^{P} z_{i, j} \hat{\mu}_{i, j}\left(x_{i}\right) \text {, }
$$

where each antecedent subset is associated with a consequent subset whose center of mass is $z_{i, j}$. Observe that the MISO IRC contains one multi-antecedent rule per element in $\mathbf{F}$, such the total number of rules is given by the product of the $N_{i}$. Conversely, the MISO URC contains one singleantecedent rule per membership vector element, giving a total number of rules equal to the sum of the $N_{i}$. Indeed, with respect to an increase in the number of antecedents, the URC achieves a linear growth in the number of fuzzy rules, while the IRC is crippled by an exponential growth in the number of rules.

In [1-3], Weinschenk et al. explore the relationship between IRC and URC fuzzy systems and prove that the IRC and the URC fuzzy inference engines given in (2) and (3) can yield identical performance if two constraints are met. First, the IRC rule table must be additively separable. A multidimensional rule table $\mathbf{F}$ is said to be additively separable if

$$
f_{a_{1}, a_{2}, \cdots, a_{P}}=\sum_{i=1}^{P} v_{i, a_{i}}
$$

where $v_{i}$ is a projection vector corresponding to the $i$ th antecedent of a multi-dimensional IRC rule table $\mathbf{F}$. Alternatively, additive seperability is expressible as

$$
f(\mathbf{x})=\sum_{i=1}^{P} v_{i}\left(x_{i}\right),
$$

for a continuous multivariate function $f$.

Secondly, equality between IRC and URC fuzzy systems requires that

$$
z_{i, j}=P v_{i, j},
$$

for all $i$. If these two constraints are met, the output formula for the URC can be written in terms of projection vectors and simplified to yield

$$
y_{U R C}(\mathbf{x})=\sum_{i=1}^{P} v_{i, j} \hat{\mu}_{i, j}\left(x_{i}\right) .
$$

When the IRC rule table is additively inseparable, a more complicated approach is required.

\section{AdDITIVELy INSEPARABLE IRC RULE TABLES}

When the IRC rule table tensor is not additively separable as described in (4), equality between the IRC and URC systems is lost. One strategy for extending the URC framework so that it may accurately implement additively inseparable IRC rule tables appeals to the field of tomography. In the field of tomography the aim is to reconstruct a surface or manifold from its projections, where each projection occurs at a different angle. Thus, reconstruction of a manifold is achieved by summing a set of filtered projections. Sets of orthogonal filtered projections, when sampled, form additively separable matrices that may be directly implemented by a URC.

Thus, an additively inseparable IRC rule table may be implemented in a layered URC architecture, where the first layer generates rotations of the inputs axes and the second layer forms the necessary filtered projections. The primary challenge is to determine which projections (at which angles) most accurately represent the rule manifold. This challenge is the converse of the problem commonly encountered in tomography where one attempts to reconstruct an unknown surface from its known projections. A tomographic approach for reconstructing a known surface with arbitrary accuracy is the focus of Subsection A. It is also necessary to develop a method for rotating the input axes within the URC framework - this is discussed in Subsection B.

\section{A. Approximation of additively inseparable rule tables}

Recall the multi-dimensional Fourier transform pair, given by

$$
F(\mathbf{u})=\int_{-\infty}^{\infty} f(\mathbf{x}) e^{-j 2 \pi \mathbf{x} \cdot \mathbf{u}} d \mathbf{x}
$$

and

$$
f(\mathbf{x})=\int_{-\infty}^{\infty} F(\mathbf{u}) e^{j 2 \pi \mathbf{x} \cdot \mathbf{u}} d \mathbf{u},
$$

where in both (8) and (9) a shorthand notation is used to express integration over the entire space as evidenced by the $d \mathbf{u}$ and $d \mathbf{x}$, respectively. Converting the integration in (9) to spherical coordinates, one obtains 


$$
f(\mathbf{x})=\int_{0}^{\pi} \int_{-\infty}^{\infty} \alpha_{\boldsymbol{\theta}}|v|^{P-1} F(v, \xi) e^{j 2 \pi v \mathbf{x} \cdot \xi} d v d \boldsymbol{\theta}
$$

where $\xi$ is a unit vector in $\mathfrak{R}^{P}$ determined by $\boldsymbol{\theta}$, and $\alpha_{\boldsymbol{\theta}}$ is the remaining portion of the Jacobian. Integration over the angles can be rewritten in terms of Riemann sums to find

$$
f(x)=\lim _{K \rightarrow \infty} \Delta \sum_{k_{1}=0}^{K-1} \cdots \sum_{k_{P-1}=0}^{K-1} \int_{-\infty}^{\infty} \alpha_{\mathbf{k}}|v|^{P-1} F\left(v, \xi_{\mathbf{k}}\right) e^{j 2 \pi v \mathbf{x} \cdot \xi_{\mathbf{k}}} d v
$$

where the angles $\boldsymbol{\theta}$ have been replaced with $K$ and $\Delta=(\pi / K)^{P-1}$.

Without loss of generality, all of the sums in (11) can be replaced by a single sum over $m$, where $m$ ranges from 1 to $M=K^{P-1}$. Further, for a fixed $\mathbf{k}$, the integral over $v$ is simply the 1-D inverse Fourier transform of a high-pass filtered version of $F\left(v, \xi_{\mathrm{m}}\right)$ along a line passing through the origin,

$$
g_{m}\left(\tau_{m}\right)=\int_{-\infty}^{\infty} \alpha_{m}|v|^{P-1} F\left(v, \xi_{m}\right) e^{j 2 \pi v \mathbf{x} \cdot \xi_{m}} d v
$$

that is oriented according to the angles given in $\left(n_{1} \pi / K, \cdots, n_{P} \pi / K\right)$. Thus, a multidimensional manifold may be represented as an infinite sum of 1-D functions as

$$
f(\mathbf{x})=\lim _{M \rightarrow \infty} \Delta \sum_{m=0}^{M-1} g_{m}\left(\tau_{m}\right) w(m)
$$

where $w(m)$ approaches unity as $M$ goes to infinity and will be discussed later. Notice for sufficiently large $M$,

$$
f(\mathbf{x}) \approx \Delta \sum_{m=0}^{M-1} g_{m}\left(\tau_{m}\right) w(m)
$$

A projection through the manifold $f(\mathbf{x})$, given by the Radon transform

$$
p(\tau)=\int_{-\infty}^{\infty} f(\tau-\xi \cdot \mathbf{x}) d \mathbf{x},
$$

is closely related to the $P$-D Fourier transform of $f(\mathbf{x})$ [14]. According to what is popularly known as the central slice theorem, the 1-D Fourier transform of a projection through a surface at a given angle is equal to a slice, taken through the origin, of the $P$-D Fourier transform at the same angle or

$$
P_{m}\left(v_{m}\right)=F\left(v_{m}, \xi_{m}\right) .
$$

Thus, through substitution of (16) into (12) we see that $g_{m}\left(\tau_{m}\right)$ is a filtered version of $p_{m}\left(\tau_{m}\right)$ which, with some manipulation, is represented in the spatial domain by

$$
g_{m}\left(\tau_{m}\right)=\alpha_{m}\left(\frac{1}{j 2 \pi}\right)^{P-1} \frac{d^{P-1}}{d \tau_{m}^{P-1}}\left\{p_{m}\left(\tau_{m}\right)\right\}
$$

for odd $P$ and

$$
g_{m}\left(\tau_{m}\right)=-2 \alpha_{m}\left(\frac{1}{j 2 \pi}\right)^{P}\left[\frac{1}{t} * \frac{d^{P-1}}{d \tau_{m}^{P-1}}\left\{p_{m}\left(\tau_{m}\right)\right\}\right]
$$

for even $P$, where $*$ represents convolution. Finally, since $g_{m}\left(\tau_{m}\right)$ is a filtered version of $p_{m}\left(\tau_{m}\right)$, and all of the 1-D $g_{m}\left(\tau_{m}\right)$ are back-projected into higher dimensions before being summed together to reconstruct the original manifold, (14) is commonly referred to as a summation of filtered back-projections in the tomography literature.

One may then define an approximation to $f(\mathbf{x})$ as

$$
\hat{f}(\mathbf{x})=\Delta \sum_{l \in \Phi} g_{l}\left(\tau_{l}\right) w(l),
$$

where $\Phi$ is a subset of $L$ indices selected from the $M$ indices given in (14). However, one must carefully choose a strategy for selecting which terms to keep from (14). Since each term kept translates to an increase in system complexity, it is important to choose a combination of the fewest possible terms to achieve a desired reconstruction accuracy. To this end, consider the following strategy for minimizing the mean-square reconstruction error.

Recall the mean-square approximation error,

$$
\text { mse }=\int_{-\infty}^{\infty}(f(\mathbf{x})-\hat{f}(\mathbf{x}))^{2} d \mathbf{x} .
$$

Substitution of (14) and (19) into (20) gives

$$
\text { mse }=\int_{-\infty}^{\infty}\left[\Delta \sum_{\substack{m=0 \\ m \notin \Phi}}^{M-1} g_{m}\left(\tau_{m}\right) w(m)\right]^{2} d \mathbf{x} .
$$

Notice that the mean square error is also the energy in a space-domain function that is composed of the 1-D filtered projections not kept in $\Phi$. Application of Parseval's theorem results in

$$
\mathrm{mse}=\int_{0}^{\pi} \int_{-\infty}^{\infty}\left[\sum_{\substack{m=0 \\ m \notin \Phi}}^{M-1} G_{m}\left(v_{m}\right) W(m)\right]^{2} d v d \boldsymbol{\theta},
$$

where $W(m)$ is desired to be unity over an $N$-D "pie-slice" defined by $m$. This imposes a windowing effect in space that 
can be made arbitrarily insignificant by increasing $M$ and will be discussed in more detail in a future publication. As the $G_{m}\left(v_{m}\right)$ are 1-D functions that lie in $P$-dimensional slices which approximate portions of the Riemann sums given in (11), the slices are disjoint. Thus, all cross terms resulting from the squaring operation must equal zero.

Therefore, when the integration over the angles is expressed in terms of approximate Riemann sums,

$$
\text { mse }=\Delta \int_{-\infty}^{\infty} \sum_{\substack{m=0 \\ m \notin \Phi}}^{M-1}\left[G_{m}\left(v_{m}\right)\right]^{2} d v
$$

However, when the integration is moved inside the sum, one finds

$$
\mathrm{mse}=\Delta \sum_{\substack{m=0 \\ m \notin \Phi}}^{M-1} E_{m},
$$

in which case the mean-square approximation error is obtained by summing the energy in the slices of the filtered $P$-D Fourier transform that are not retained in $\Phi$. Therefore the mean-square reconstruction error is minimized for a fixed amount of resources by choosing to keep those terms with the most energy.

In order to proceed, a transform is needed to efficiently compute the energy in all possible slices through the origin of the $P$-D Fourier transform. This is accomplished with the $\mathrm{X}$ Ray transform given by

$$
\tilde{f}(\rho, \boldsymbol{\theta})=\int f(\mathbf{x}) \delta(\rho-\xi \cdot \mathbf{x}) d \mathbf{x}
$$

where $\rho$ is the distance from the origin and $\xi$ is a unit vector that gives the orientation of a line. However, since we are only interested in those (filtered) slices of $F$ that pass through the origin, $\rho$ is set to zero.

As will be discussed in the next subsection, the resources required to rotate the inputs axes are minimal as this operation simply amounts to a weighted sum and need not even be performed by a fuzzy layer. Neglecting these resources, one finds the complexity of the layered URC scales with the number of rules in the second URC layer given by $N L$. In Section II, the rules contained in an IRCbased system are given as the product of the numbers of antecedent subsets or $N^{P}$. Thus, the rule savings offered by the layered URC is given as

$$
\text { Complexity Savings }(\%)=\frac{L}{N^{P-1}} \times 100 \text {. }
$$

Thus, when the number of projections required is moderate, the complexity savings is exponential.

\section{B. Rotation of the input axes}

Once the 1-D functions $g_{m}\left(\tau_{m}\right)$ are selected, the input axes must be rotated to accommodate the desired projections as shown in Fig. 1. This rotation is implemented by

$$
r_{\theta}=\mathbf{x} \cdot \xi
$$

where, once again, $\xi$ is a unit vector that indicates the orientation of the new axis. Conveniently, the rotated input given in (27) is additively separable as defined in (5), where

$$
r_{\theta}=\sum_{i=1}^{P} x_{i} \xi_{i}
$$

Thus, (28) is easily implemented in a URC because the URC output formula may be written as

$$
y_{U R C}(\mathbf{x})=\sum_{i=1}^{P} s_{i}\left(x_{i}\right)
$$

where

$$
s_{i}\left(x_{i}\right)=\sum_{j=1}^{N_{i}} v_{i, j} \mu_{i, j}\left(x_{i}\right) .
$$

Thus, membership functions may be interpreted as interpolation kernels such that the function $s_{i}\left(x_{i}\right)$ is an interpolated version of $x_{i} \xi_{i}$, where the elements of the projection vectors $v_{i, j}$ are samples of $x_{i} \xi_{i}$. Indeed, it is possible to perform any type of Lagrange interpolation by choosing the membership functions to be piecewise defined from sections of the Lagrange interpolating polynomials. Other types of interpolation are also possible.

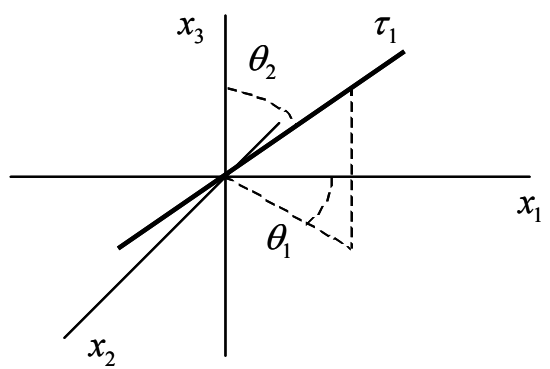

Fig. 1. The function $g_{1}\left(\tau_{1}\right)$ operates on the variable $\tau_{1}$ which is oriented as shown with respect to the input axes $x_{1}$ and $x_{2}$.

\section{IMPLEMENTATION ISSUES}

Naturally, one may use the integral transforms given above to directly compute the optimum 1-D functions and their corresponding orientations in space for a given $P$-D manifold whose behavior is known over the entire space. However, when the $P$-D manifold with infinite support is artificially truncated (or windowed) such that the 1-D functions may be 
computed using a discrete mathematics software package, the process becomes slightly more involved. Two such procedures are summarized below.

First, one may elect to directly compute the $P$-D FFT on a rectangular grid of samples from the desired manifold. However, the implementation of this method requires the use of a circular (or circularly symmetric) window in order to prevent the transform from being unnecessarily biased by the long diagonals of the rectangular grid. Next, the Fourier transform is filtered according to (12) followed by computation of the energy in each slice (via the X-ray transform) in order to select the optimum 1-D functions and their orientations. Once the optimum slices of the $P$-D Fourier transform have been identified, an approximate manifold may be constructed. However, this approach is somewhat undesirable as it is necessary to interpolate in the frequency domain - a procedure that can unnecessarily introduce a great deal of error.

Alternatively, one may compute spatial projections through the $P$-D manifold via the radon transform given in (15). Recall these projections are related to slices of the $P$-D Fourier transform through the Fourier slice theorem discussed previously. Thus, these projections may be filtered in space by (17) or (18), or in frequency by (12). Next, the energy per slice is computed in order to select the optimum 1-D functions and function orientations. In this method, interpolation is only performed in the spatial domain, which should keep errors to a minimum as it is presumed that the manifold is well known over the region of interest.

When the manifold is only known over a rectangular grid of points, the projections through the surface must be carried out in the manner demonstrated below (in 2-D) in Fig. 2. In Fig. 2, a 2-D surface is accurately known on an $a \times a$ region of space. In order to prevent the square window from biasing the projections, projections must be computed from equalarea square regions that lie within the $a \times a$ grid. Naturally, when reconstructing the manifold from filtered versions of these projections, the reconstruction is only accurate in that region where all of the projections overlap - the innermost dashed circle in Fig. 2. Therefore, the largest grid that may be accurately reconstructed from the original set of samples is of dimensions $a / 2 \times a / 2$ as shown.

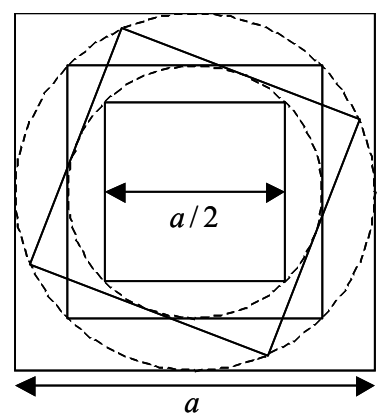

Fig. 2. Computation of projections from a rectangular gird of samples.
Now let us consider a specific 2-D example. We choose an XOR-shaped surface such that these results may be easily connected to our previous findings in other publications. In particular, we approximate the additively inseparable surface given by

$$
f\left(x_{1}, x_{2}\right)=-2 x_{1} x_{2} .
$$

Note that this surface is additively separable when rotated by 45 degrees, as

$$
f_{45^{\circ}}=x_{1}^{2}-x_{2}^{2} \text {. }
$$

Projections are computed for 180 angles (in degrees) evenly spaced on the interval $[0,179]$. These projections are then filtered in the frequency domain according to (12). Finally, the energy in each of these slices is computed and plotted in Fig. 3. Notice that the filtered projections with the most energy are those corresponding to angles of 45 and 135 degrees, as one would expect given (32). The filtered projections obtained at 45 and 135 degrees are shown along with their ideal counterparts in Fig. 4. Notice that the estimates are most accurate in the center. This is a direct result of the filtering as the projection is convolved in space with the reciprocal of $\tau$, as described in (18). Thus, the points closer to the edges of the rectangular window are less accurate as the convolutions attempt to bring in non-zero portions of (31) that are implicitly defined to be zero by the rectangular window.

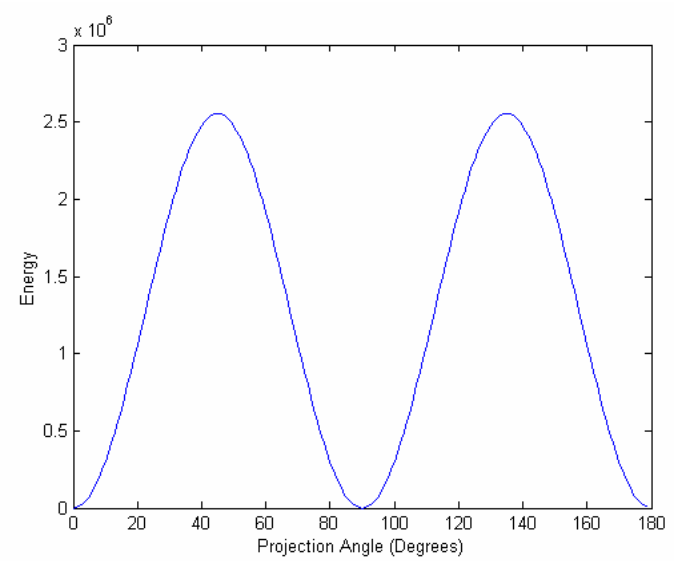

Fig. 3. Energy per filtered projection vs. function orientation (projection angle).

The original surface from (31) is shown in Fig. 5. The reconstructed surface is not shown as it is visually indistinguishable from Fig. 5. However, the absolute error per sample is shown in Fig. 6. Notice that, as indicated in Fig. 4, the most significant error occurs on the periphery. The overall mean-square error for the reconstruction is $6.25 \times 10^{-5}$ percent per sample. 


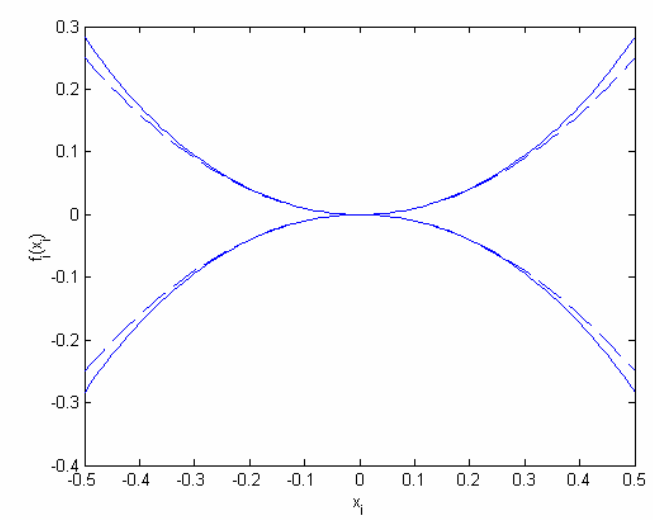

Fig. 4. Estimated filtered projections for the desired surface given in (31) at angles of 45 and 135 degrees. The actual filtered projections are shown in dashed lines.

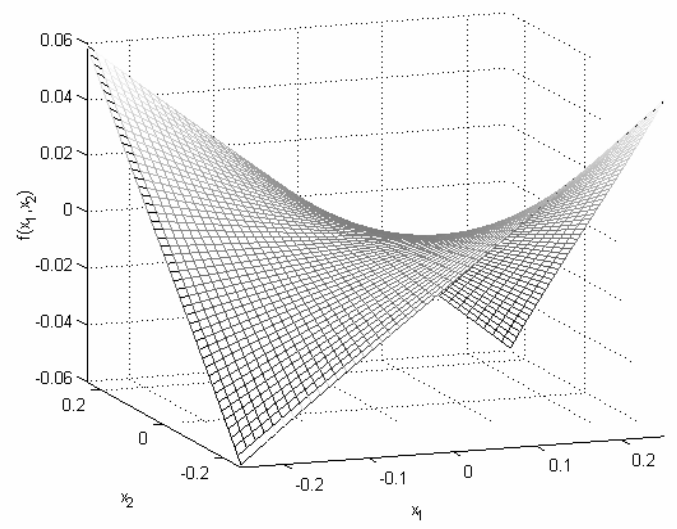

Fig. 5. Central portion of the original XOR surface. The reconstructed surface is visually indistinguishable from this figure.

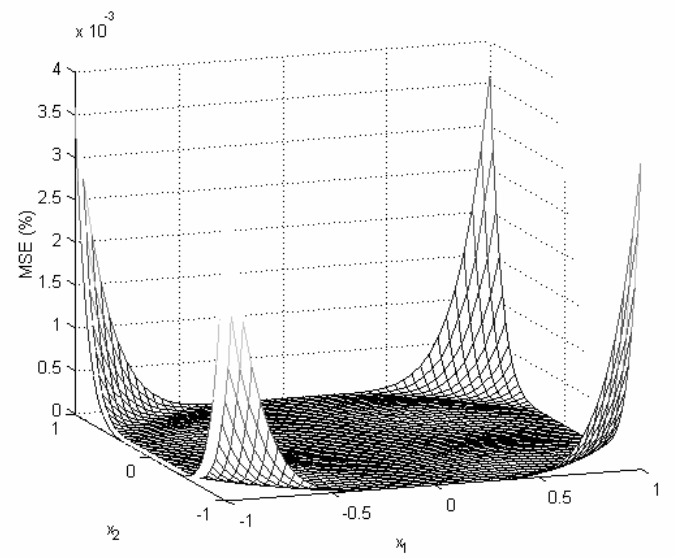

Fig. 6. Mean-square error, per sample (in percent).

Finally, a 2-layer URC fuzzy system is constructed, the second layer of which is constructed from samples of the filtered projections shown in Fig. 4. Further, an IRC system is also constructed for comparison purposes. However, the output surfaces for both the URC and IRC fuzzy systems are visually indistinguishable from that shown in Fig. 5 and, due to a lack of space, are not shown. The mean-square error per sample is $8.64 \times 10^{-5}$ percent. Thus, the URC accurately captures the IRC functionality while, importantly, the URC retains its exponential complexity savings. For a further discussion of the resulting complexity reduction, please consult [3].

\section{CONCLUSION}

We have developed a method for constructing URC fuzzy systems from IRC fuzzy systems that have an additively inseparable rule table. This Fourier-based approach relies on tomographic principals to determine those projections through the desired rule manifold that will provide, for a fixed amount of resources, an optimal reconstruction in the minimum mean-square reconstruction error sense. This approach allows one to achieve (in many cases) an exponential reduction in complexity with arbitrary reconstruction accuracy where the tradeoff between reconstruction accuracy and amount of resources is easily adjusted.

\section{REFERENCES}

[1] J. J. Weinschenk, W. E. Combs, R. J. Marks II, "Avoidance of rule explosion by mapping fuzzy systems to a union rule configuration," Proc. of the FUZZ-IEEE, pp 43-48, 2003.

[2] J. J. Weinschenk, R. J. Marks II, W. E. Combs, "Layered URC fuzzy systems: a novel link between fuzzy systems and neural networks," Proc. of the Intl' Joint Conf. Neural Nets, pp 2995-3000, 2003.

[3] J. J. Weinschenk, R. J. Marks II, W. W. Combs, "On the avoidance of rule explosion in fuzzy systems," forthcoming from IEEE Transactions on Fuzzy Systems.

[4] W. E. Combs and J. E. Andrews, "Combinatorial rule explosion eliminated by a fuzzy rule configuration," IEEE Trans. Fuzzy Systems, vol. 6, no. 1, pp. 1-11, Feb. 1998.

[5] J. Yi, N. Yubazaki, and K. Hirota, "A proposal of SIRMs dynamically connected fuzzy inference model for plural input fuzzy control," Fuzzy Sets and Systems, vol. 125, pp. 79-92, 2002.

[6] J. M. Mendel and Q. Liang, "Comments on "Combinatorial rule explosion eliminated by a fuzzy rule configuration," IEEE Trans. Fuzzy Systems, vol. 7, no. 3, pp. 396-373, June 1999.

[7] W.E. Combs, "Author's Reply," IEEE Transactions on Fuzzy Systems, vol. 7, no. 3., pp. 371-373, June 1999.

[8] S. Dick and A. Kandel, "Comment on "Combinatorial rule explosion eliminated by a fuzzy rule configuration," IEEE Trans. Fuzzy Systems, vol. 7, no. 4, pp. 475-477, Aug. 1999.

[9] W.E. Combs, "Author's Reply," IEEE Trans. on Fuzzy Systems, vol. 7, no. 4., pp. 477-478, Aug 1999.

[10] E. Trillas and C. Alsina, "On the law $[\mathrm{pAq}->\mathrm{r}]=[(\mathrm{p}->\mathrm{r}) \mathrm{V}(\mathrm{q}->\mathrm{r})]$ in fuzzy logic," IEEE Trans. on Fuzzy Systems, VOL. 10, NO. 1, pp. 8488, Feb 2002.

[11] B.-S. Chen, H.-J. Uang, and C.-S. Tseng, "Robust Tracking Enhancement of robot systems including motor dynamics: a fuzzybased dynamic game approach," IEEE Trans. Fuzzy Systems, Vol. 6, pp 538-552, Nov 1998.

[12] H. Emara and A. L. Elshafei, "Comments on 'Robust Tracking of robot systems including motor dynamics: a fuzzy-based dynamic game approach", IEEE Trans. on Fuzzy Systems, Vol. 10, No. 3, pp. 412414, June 2002.

[13] B.-S. Chen, H.-J. Uang, and C.-S. Tseng, “Author's Reply", IEEE Trans. on Fuzzy Systems, Vol. 10, No. 3, pp. 414, June 2002.

[14] A. G. Ramm and A. I. Katsevich, The Radon Transform and Local Tomography, CRC Press, 1996. 